\title{
Public Health Information Management System at MOH level
}

\author{
Dr. E.S.S. Rodrigo \\ Postgraduate Institute of Medicine, University of Colombo, Sri Lanka. \\ E-mail address: semuthurodrigo@gmail.com
}

eHealth Sri Lanka 2010,1(suppl.1):S2

DOI: http://dx.doi.org/10.4038/sljbmi.v1i0.3531

Only the Abstract is available

\begin{abstract}
The preventive care in Sri Lanka considerably depends on the grass root level of public health carried out by Public Health Midwives and Public Health Inspectors (PHMM \& PHII) who are working under Medical Officers of Health working across the country. In addition, all the final statistics at central level are generated using these data provided by the above field workers via the $\mathrm{MOH}$ offices. Therefore, it is important to collect and maintain accurate data at this level.

This paper introduces a Public Health Information Management System that facilitates storing and manipulating data of residents, who are at the relevant $\mathrm{MOH}$ areas. There is an advanced search mechanism in this system which gives many outputs. Thus, it is useful for the administrative officers and the other public health staff to get a clear view of the health problems in the area and to attend to them. Usefulness of the system will be greatly increased if real time data, collected by means of surveys are entered in the system.

The database should be updated regularly using the information provided by the field workers. Database is located in the $\mathrm{MOH}$ office and it is possible to upload data to a central database when required.

This system facilitates monitoring and evaluating field activities of PHMM and PHII by the supervisors and field workers themselves. In addition, it is possible to generate several routine reports and returns that are sent to the regional and central offices. This reduces the time wastage of PHMM/PHII in paper work, giving them the opportunity to involve in more field activities. The system provides the capability of storing maps of the area and gives the facility to mark index cases in communicable and non communicable disease surveillance and maternal and child health activities.
\end{abstract}

Keywords - PHMM \& PHII, facilitates storing, real time data, disease surveillance, 